\title{
A Survey on Determining Urban Emotions using Geo-Data Classification: A Case Study around Majitar, East District, Sikkim
}

\author{
Supriya Choudhury \\ Sikkim Manipal Institute of \\ Technology \\ Majitar, Rangpo-737136, \\ East District, Sikkim
}

\author{
Mohan P. Pradhan \\ Sikkim Manipal Institute of \\ Technology \\ Majitar, Rangpo-737136, \\ East District, Sikkim
}

\author{
S. K. Kar \\ Sikkim Manipal Institute of \\ Technology \\ Majitar, Rangpo-737136, \\ East District, Sikkim
}

\begin{abstract}
Perception and expectation of citizens is an important factor in urban planning, settlement and management. Hence, there is a need of a participatory citizen centric planning of urban settlement based on spatial data. These perception and expectation may be represented in terms of emotions. Determining Urban Emotions is an approach which can be used to map different types of emotions associated with urbanization. In the recent years, some new methods have been presented for the area of urban and spatial planning, resulting in a fundamental change of the understanding of urban planning. Geographical information system acts as a key factor for analyzing urban emotions from various types of data. This paper presents the review of ongoing research on various techniques for determining urban emotions in the recent years.
\end{abstract}

\section{Keywords}

Urban Planning, Spatial planning, Smart city, People as Sensors, Urban Emotions, Spatial monitoring, Psychophysiological monitoring, Mental Maps, Psycho-geography, Bio-mapping, Emotional cartography.

\section{INTRODUCTION}

Urban Emotion is one of the emerging approaches that combine not only the spatial planning and geographic information systems, but also concepts of computer linguistics, sensor technology methods and real world data. Spatial planning considers all social and spatial structures within the city and helps in collecting various forms of data in context to the city. It involves both the spatial and temporal patterns that help in research activities in identifying processes and to characterize special social-cultural movements and developments. Geographic information system consists of two distinct disciplines geography and information system. It is an information system that is designed to work with data referenced by spatial or geographical coordinates. Computer linguistics is an interdisciplinary field concerned with the statistical or rule based modeling of natural language from a computational perspective. Real world data is an umbrella term used for different types of data that are collected in conventional randomized controlled trials. It can be technical sensor data, crowd sourced data, human- sensor data or social data, etc. It can be used for decision making.

The main idea behind this approach is the involvement of people of a particular location into various planning processes. Urban Emotion deals with different expectations of people regarding a particular location and what additional features can be added to the locality. It explains the potential of integrating objectively quantified emotions in context of citizen participation. Determination of Urban Emotion figures out the use of real world data.

Citizen's perception and urban space when linked together triggers an emotional reaction and creates its own atmosphere in the observer. Urban emotion aims to understand how people's feelings get affected by features of the current environment, green spaces, air pollution, water pollution, noise pollution, affects of industrialization, land degradation, road condition, and other geographical factors.

Better urban planning approaches are needed to build a city into a smart city. Smart cities are the cities that are able to operate in a sustainable, efficient and intelligent manner and require smart infrastructure with advanced sensing capabilities that extend beyond mere technical subtitles, thereby possibly benefitting architects and citizens of the cities. It means smart citizens can make intelligent cities. It relies on the idea that only citizens can make a city really intelligent. It needs to be tackled both from technological view point and human centric view point that a city requires smart citizens to be intelligent themselves.

There are two types of monitoring approaches used for determining Urban Emotions-

1. Spatial monitoring approach [1] and

2. Psycho-physiological monitoring approach [1] [2]

Spatial monitoring approach [1] includes sensor driven data gathering and human sensory assessment. Further, there are two types of spatial monitoring approach-

a) Deductive monitoring and

b) Inductive monitoring.

Deductive monitoring [1] can be described as the observation of phenomena over a time span, which may be integrated in a Geo-web-supported planning process and can be organized by planning institutions who are interested in the gathered topic. It is used in top-down planning mode. It is a top-down approach which is mainly top-down oriented and composed of data generated by time series analysis. Deductive monitoring system is initiated by a public or private authority in a topdown process with specific task.

Inductive monitoring [1] can be described as an expression of crowd-sourcing processes in bottom up planning mode. In inductive monitoring system, no fixed top-down oriented infrastructure is given. Expressions and statements regarding a special topic are collected without being initiated by a traditional, organizationally driven top-down process.

Psycho-physiological monitoring [1] [2] [7] is a technology used to measure the arousal of the citizens. It provides data about the change in body reactions (physiology) of the 
citizens [2]. It refers to the gathering of bio-sensor, biostatistical and physiological data. For example, measuring the startle reflex of the body, reflex of neck or the eye, etc.

\section{RELATED WORK}

The problems of considering subjective measurements and views provided by the citizens by involving them into planning processes represent great challenges for efficient urban planning. The wide range of the problems of extracting human emotion in context to the city may make good understanding of different expectation of the people. Urban Emotion adds a new information layer which will help in urban planning. Many researchers had created several methods to deal with the problem of determining urban emotions. In this paper, we managed to review and summarize the major techniques for determining urban emotions. We categorized some of these techniques based on the various approaches which the technique is using.

An Urban Emotion provides a human-centered approach for extracting contextual emotion information by using the concept of People as Sensors, where the data provided by the people may be in the form of their social activities like Twitter data, or in the form of their usage of an smart phone app, else may also be a real-time data which we may obtain from the people residing in a particular area in the form of their answer to certain predefined queries.

In earlier approaches, the different aspect of human emotion in spatial planning and urban space is linked with perception in planning, mental maps and psycho-geography. Mental Maps [1] [2] tries to analyze the quality in a physical object which gives it a high probability of evoking a strong image in a given observer. The study of human emotion using mental map is basically known from Kevin Lynch and the research of Image of the City. Psycho-geography [1] is a term applied to an inventive strategies used for exploring cities, which includes anything that takes citizens off their predictable paths and include them into a new awareness of the urban landscape. The study of human emotion using psychogeography came into existence during the urban transformation process in Paris in the year 1950. The determination of Urban Emotion figures out the use of technical sensor data and human sensor data. The technical sensor data is obtained in the context of measurements from calibrated bio-sensors whereas; the human sensor data is obtained in the context of subjective observations by citizens residing in that particular region.

Peter Zeile et al. in [1] introduced the research field named Urban Emotions based on mental maps and psycho-geography in combination with the field of digital emotion based urban tagging. New Bio-statistical and Sensor technology were used for understanding the concept of Smart City, People as Sensors and Urban Tagging. A psycho-physiological measurement in urban space was used to map emotions. Smartphone data and social media data has been used to get collective human behavior patterns. A RADAR Sensing concept and app for Android OS was developed. User's position was detected using multiple sensors (preferably GPS) and displaying the position on a map. Emotions and emotion information were extracted from Volunteered Geographical Information (VGI) and from user-generated information, such as Twitter, Flickr or Facebook posts. A label propagation algorithm was created using methods of geo-informatics and computational linguistics where concepts of distances, clusters and similarities exist in both domains and can be combined natively in a graph-based algorithm, which were further used to identify emotions in tweets and classify them into appropriate categories. Bio-statistical data set in relation to the collected subjective feelings has been used to get qualitative information about the sensor detected arousal or perception of the test person. Statement of Emotion, detecting the potential spatial context and rating the intensity of emotion and Notification (time based, location based, trigger based) has been used to give a rating of the personal perception or emotion.

The advantage of this approach was better identification of trigger of an emotion and analysis of the intensity of emotion. There was automatically tagging of place and time and transmission to the server. Visualizations (in near real time) were used to get a visual feedback. Negative emotions were identified as "stress" by measuring a combination of body temperature and skin conductance.

The disadvantage of this approach was the availability of no real time sensor technology. A systematical evaluation of spatial or planning relevant issues was not realized. The methods based on a subjective rating of a statistical situation and the test persons were not in situ. There was no embedding of people, and the situation-based urban context was also not considered. The user was permanently in a kind of conflict between participative motivation and concern for privacy. The danger was that users do not begin or end participation due to their fear that data may be accessed by other users or even companies which was a huge barrier. It was less about technical hurdles, but rather to raise awareness of the user of the dangers, but also the potentials for the participation in city and urban planning.

In [2], both technical and human sensors along with georeferenced social media posts, was used to extract context based emotional information from them for collecting and analyzing data on the basis of emotional perception to urban space [2]. The privacy issues associated with the data and the potential of the wearables were discussed. Human sensor network was created by merging and combining the objectively quantified elements of sensor technology with the subjective measurement methods. The concepts of Mental Maps or Cognitive Maps are used as main component and for cartographic representation. Analog techniques like sketches or descriptions were combined with digital methods. Various methods like Bio-mapping technique and Emotional Cartography were used. GPS, microphones and cameras were the basic requirements of participatory sensing and Smart phones were the most efficient standard sensing with integrated sensors. Wearables were used to measure physiological data like skin conductance, skin temperature or electrocardiography (ECG). Emotions were detected using wristband sensors and emotion information was extracted from user-generated data, crowd-sourced data and geo-social media like Twitter, that is, from unstructured datasets such as tweets. Text processing algorithms was used for preprocessing tweet text. The similarity was computed between all tweets. A tweet graph was constructed, where thicker edges between tweets indicate higher similarity scores. A graph-based label propagation algorithm was used. Tweets were labeled in a semi-supervised learning approach. Urbantagging was done by using the smart phone RADAR SENSING app. They used People as Sensors smart phone app for ground-truthing emotion measurements and Emotion Sensor to detect spikes in different biometric parameters. The Concept of Psycho-physiological monitoring was helpful in identifying emotions using emotional stimuli with IAPS (International Affective Picture System). For example, the measurements of startle reflex. The methods like barrier free 
planning was used for rating accessibility of infrastructures. A temporal barrier or only a personal negative impression was helpful in identifying if there really is a planning deficiency. They used the concept of emocycling and summarized some of the risks and limits of using physiological data.

Some of the limitations [2] of using physiological data [7] are given as follows:

a. Data privacy and protection [2].

b. Lack of clarity, commercialization and information overload must be monitored [1].

c. Technical protection mechanisms, citizen's awareness for transparency, openness and especially control over data all are important.

d. The use of personal data should be well documented.

e. Problem is to raise awareness of the multidimensional connections more than about the technical hurdles.

The disadvantage of this approach was that there has been still a scientific lack in the discussion of emotional aspects in urban planning. The use of emotions in urban design was not well-established and far grown. Urban Emotions was not conceived as a generalized tool of solving all types of planning tasks, but it may help to create another view and provide a better understanding of the body of the city. Urban Emotions lacked in providing valuable information in every case. The demands for data protection are even higher. It bought users into permanent conflict between participative motivation and concerns for their own privacy. The postulated freedom in the internet was equally compromised by commercial companies. The problem was to raise awareness of the multi-dimensional connections.

In [4], the concept of Smart City was defined. The emotion information was used in urban planning. The emotion data were collected through surveys and interviews. A direct feedback from citizens via People as Sensors app or eDiaries was provided to stakeholders and participatory communication channels like Citizen Sensing were used in real time. Human sensor network was created. The mobile applications were used for collecting physiological data. Physiological measurements were used in urban planning. Data that was collected were measured by a wearable device named biosensor. Subjective feelings of people were collected using questionnaires and conventional diaries. The concept of Biomapping, Crowd-sourcing physiological and subjective emotions was discussed. Mapiness app, traditional deductive planning and inductive planning approaches were being used. Crowd-sourcing emotion information was done via technical and human sensors. Assessing emotion has been assessed using both technical sensors and human sensors. A Smartphone app named as People as Sensors, trigger, a pluggable module, and a service-oriented architecture were further been used.

The advantage of this approach was that they analyzed personal data anonymously (that is, in the form of an aggregated data rather than individual datasets) and tried to provide a new layer of information for urban planning processes. Also, users were able to immediately compare their own perceptions and impressions with other people.

The disadvantages of this approach were the methods were somewhat complicated, prone to error, and were not reliable. The data used to extract human emotions were near real time. The creation and analysis of questionnaires were difficult. Instant on-site collection of data using questionnaires was virtually impossible. As a result, it did not give a conclusion that emotional information can be transferred directly into categorical values and used for urban planning. Measuring emotions with the help of psycho-physiological sensors was still challenging from a psychological and a physiological viewpoint. The major risk was that the exact information about the location of an event may get lost and sometimes the location information was difficult to resemble.

In [9], they examined urban patterns and city structures regarding the changing needs of people in their everyday life. Ubiquitous Computing and pervasive sensing with mobile devices which can act as small and connected sensors in the spatial environment were used. RADAR SENSING app and RADAR infrastructure were used as a real-time monitoring tool. The deductive and inductive monitoring approaches were further been used.

Bernd Resch in [8] used ubiquitous sensor networks and location-based services. He introduced the concept of People as Sensors, Collective Sensing, Citizen Science, and Participatory Sensing. He used Redwood Watch locationbased application and OpenSensTracker application. He did the comparison between the concepts of People as Sensors, Collective Sensing and Citizen Science. Live Geography infrastructure system and open standards such as the Open Geospatial Consortium (OGC) Sensor Observation Service (SOS) was used which allows for simple integration of usergenerated observations into geospatial data analysis and visualization systems via pull-based mechanisms (OGC SOS, Keyhole Markup Language-KML or GeoRSS) or via push based alerting services (OGC Sensor Alert Service-SAS, OGC Sensor Event Service-SES or Common Alerting ProtocolCAP). The sending of data to the Geo-Tracking \& Fusion Server was done by a simple HTTP GET request containing the measurements as GET key-value pairs. Data was integrated via a transactional instance of the Sensor Observation Service (SOS-T). He collected data from Volunteered Geographic Information (VGI). He used Infrastructure-oriented approaches and directives such as Global Monitoring for Environment and Security (GMES), Infrastructure for Spatial Information in Europe (INSPIRE) and the Shared Environmental Information Space (SEIS).

The major disadvantages with this approach were the creation of a standardized measurement infrastructure using well conceived data and service standards that was a major technical challenge, Consistent semantic encodings required standardization on two levels - firstly on sensor data level (encodings for measurements) and secondly on phenomenon level (measured encodings). Representativeness in VGI was a connected methodological issue in the field of semantics.

Bernd Resch et al. in [3] introduced an interaction model of smart city with context factor as the common interface between humans, the environment, and technology. They discussed both the unintentional and the intentional sensing capabilities of different technologies and how the different sensors were being used to collect context based information for sorting and analysis purpose. They defined external (or physical) contexts, Internal (or cognitive) contexts and Spatiotemporal contexts. The focal premises were used. Noise was measured to generate dynamic noise maps for spatial planning purposes. Context-aware analysis approach and an adaptive geo-monitoring framework were further used. They used the concept of information fusion, the Internet of Things (IoT), smart home ( $\mathrm{SH})$ technology and contextual sensing. Geospatial information acted as the key to indicator-based performance analysis in urban environments. 
The disadvantage with this approach was direct technological sensing of human-environment interactions was not possible. Human-environment interactions were highly complex, multifaceted, very poorly represented by some sort of proxy data and were not represented in objective electronic sensor data from calibrated hardware. Analytical smartness in solving on site problems was partly outsourced or decentralized. The use of remote sensing technologies in smart city applications was limited.

Chunnu Khawas et al. in [10] performed an evaluation of Land coverage and utilization pattern. They introduced the concept of urban sprawl. Various patterns of sprawling were discussed. Base map was generated using ERDAS 9.4 tool kit. Thematic vectors were created. The concepts of satellite image classification and maximum likelihood classification technique was used. Population growth was calculated using predictive approach. Growth rate and exponential growth was also calculated. Extend and trend of sprawling pattern, area of interest, type and direction of sprawling was identified.

The previous approaches for determining Urban Emotion consists of four steps summarized mainly as,

Step1: Detecting human emotions using wristband sensors [1]

[2] [5], that is, wearables [1] to measure citizen's bio feedback in context of urban areas.

Step2:Ground-truthing measurements [1] [2], that is, assigning a formal emotion category to each measurements using the concept of People as Sensors [9] location based service [5] [8] or location aware Smartphone (mobile) application. For example, using RADAR SENSING app, this locates the citizen's (or user's) position directly with the help of various sensors like GPS and WIFI. Also, by geo-tagging or urban tagging based positive or negative impression.

Step3: Extracting human emotions from the crowd-sourced data [6] and geo social media like Twitter [5] [2], this is done similar to the sentiment analysis [5]. In this step, a semi-supervised learning algorithm (approach) is used, which can be applied to a dataset with few labeled, but numerous unlabelled instances (a smaller data set), and evaluated can also be used on a test set.

Step4: Correlating the extracted and measured human emotions in order to provide urban planners with additional insights into the complex human-city relationship [2]. In this step, the human emotion is mapped with the help of bio-statistical data. Thus, the contextual emotion information extracted can serve as the citizen's direct feedback for urban planning, decision support, evaluating ongoing planning and design processes.

\section{CONCLUSION}

In the recent years, various techniques have been gained popularity because it provided some new approach for determining urban emotion. However, it has been observed that every technique has its own advantage and disadvantages. The integration of real world data and geographical-data helps in determining various ways which are to be considered and suggested for better urban planning. Thus, Urban Emotions act as new information layers within planning processes [4]. Hence, determining Urban Emotions using Geo-Data classification helps in understanding urbanization and in urban planning. Urban emotion is becoming one of the major areas of research that aims at quality planning and urban settlement prior to its implementation for the betterment of citizens and humanity at large. It tries to get more beneficial than the various techniques earlier used and at the same time it also tries to remove the individual disadvantages of each of the techniques by mitigating them on common features.

\section{REFERENCES}

[1] Peter Zeile, Bernd Resch, Linda Dorrzapf, Jan-Philipp Exner, Gunter Sagl, Anja Summa, Martin Sudmanns, Urban Emotions-Tools of Integrating People's Perception into Urban Planning, Conference Proceedings REAL CORP 2015 Tagungsband, 5-7 May 2015, Ghent, Belgium. ISBN: 978-3-9503110-8-2 (CD-ROM); ISBN: 978-3-9503110-9-9 (Print).

[2] Peter Zeile, Bernd Resch, Jan-Philipp Exner and Gunther Sagl, Urban Emotions Benefits and Risks in Using Human Sensory Assessment for the Extraction of Contextual Emotion Information in Urban Planning, Springer International Publishing, 2015.

[3] Bernd Resch, Martin Sudmanns, Gunther Sagl, Anja Summa, Peter Zeile, and Jan-Philipp Exner, Crowdsourcing Physiological Conditions and Subjective Emotions by Coupling Technical and Human Mobile Sensors, GI_Forum - Journal for Geographic Information Science, 1-2015, Berlin, ISBN 978-3-87907558-4, ISSN 2308-1708, doi:10.1553/giscience2015s514

[4] Gunther Sagl, Bernd Resch, and Thomas Blaschke, Contextual Sensing: Integrating Contextual Information with Human and Technical Geo-Sensor Information for Smart Cities, Open Access Sensors, 2015, 15, 1701317035; doi: 10.3390/s150717013, ISSN 1424-8220.

[5] Bernd Resch, Anja Summa, Gunther Sagl, Peter Zeile, Jan-Philipp Exner, Urban Emotions-Geo-Semantic Emotion Extraction from Technical Sensors, Human Sensors, Springer International Publishing, 2014.

[6] Chrysaida-Aliki Papadopoulou and Maria Giaoutzi, Crowd-sourcing as a Tool for Knowledge Acquisition in Spatial Planning", Future Internet 2014, 6, 109-125; ISSN 1999-5903, doi:10.3390/fi6010109.

[7] Benjamin S. Bergner, Jan-Philipp Exner, Martin Memmel, Rania Raslan, Dina Taha, Manar Talal, Peter Zeile,"Human Sensory Assessment Methods in Urban Planning - a Case Study in Alexandria", Conference Proceedings REAL CORP 2013, Tagungsband, 20-23 May 2013, Rome, Italy, ISBN: 978-3-9503110-4-4 (CDROM); ISBN: 978-3-9503110-5-1 (Print).

[8] Bernd Resch, "People as Sensors and Collective SensingContextual Observations Complementing Geo-Sensor Network Measurements", Springer International Publishing, 2013.

[9] Peter Zeile, Martin Memmel, Jan-Philipp Exner, "A New Urban Sensing and Monitoring Approach: Tagging the City with the RADAR SENSING App", Reviewed Paper of Conference Proceedings REAL CORP 2012, Tagungsband, 14-16 May 2012, Schwechat, ISBN: 9783-9503110-2-0 (CD-ROM); ISBN: 978-3-9503110-3-7 (Print).

[10] Chunnu Khawas, Mohan P. Pradhan, M. K. Ghose, "A Statistical Approach to Urban Sprawling using RS \& GIS - A Case Study of East District, Sikkim”, Proceedings published by International Journal of Computer Applications (IJCA), 2011. 